\title{
Defining Agents' Behaviour for Negotiation Contexts
}

\author{
João Carneiro $^{1}$, Diogo Martinho ${ }^{1}$, Goreti Marreiros ${ }^{1}$ and Paulo Novais ${ }^{2}$ \\ ${ }^{1}$ GECAD - Knowledge Engineering and Decision Support Group, Institute of Engineering - \\ Polytechnic of Porto, Porto, Portugal \\ \{jomrc, 1090557, mgt \} @ isep. ipp.pt \\ ${ }^{2}$ CCTC - Computer Science and Technology Center, at University of Minho, \\ Braga, Portugal \\ pjon@di.uminho.pt
}

\begin{abstract}
Agents who represent participants in the group decision-making context require a certain number of individual traits in order to be successful. By using argumentation models, agents are capable to defend the interests of those who they represent, and also justify and support their ideas and actions. However, regardless of how much knowledge they might hold, it is essential to define their behaviour. In this paper (1) is presented a study about the most important models to infer different types of behaviours that can be adapted and used in this context, (2) are proposed rules that must be followed to affect positively the system when defining behaviours and (3) is proposed the adaptation of a conflict management model to the context of Group Decision Support Systems. We propose one approach that (a) intends to reflect a natural way of human behaviour in the agents, (b) provides an easier way to reach an agreement between all parties involved and (c) does not have high configuration costs to the participants. Our approach will offer a simple yet perceptible configuration tool that can be used by the participants and contribute to more intelligent communications between agents and makes possible for the participants to have a better understanding of the types of interactions experienced by the agents belonging to the system.
\end{abstract}

Keywords: Group Decision Support Systems, Ubiquitous Computing, Affective Computing, Multi-Agent Systems, Automatic Negotiation

\section{Introduction}

Rahwan et al. (2003) defined negotiation as "a form of interaction in which a group of agents, with conflicting interests and a desire to cooperate, try to come to a mutually acceptable agreement on the division of scarce resources" [1], Hadidi, Dimopoulos, and Moraitis (2011), defined negotiation as "the process of looking for an agreement between two or several agents on one or more issues" [2] and El-Sisi and Mousa (2012) defined as "a process of reaching an agreement on the terms of a transaction such as price, quantity, for two or more parties in multi-agent systems such as ECommerce. It tries to maximize the benefits to all parties" [3]. It is possible to verify 
in the literature a consensus regarding to the main approaches to deal with negotiation: game theory, heuristics and argumentation [1-3]. It is a known fact that game theoretic and heuristic based approaches evolved and turned more complex. With this development they have been used in a wide range of applications. However they share some limitations. In the majority of game-theoretic and heuristic models, agents exchange proposals, but these proposals are limited. Agents are not allowed to exchange any additional information other than what is expressed in the proposal itself. This can be problematic, for example, in situations where agents have limited information about the environment, or where their rational choices depend on those of other agents. Another important limitation is that agent's utilities or preferences are usually assumed to be completely characterized prior to the interaction. Thus, to overcome these limitations, argumentation-based negotiation appeared and turned one of the most popular approaches to negotiation [4], it has been extensively investigated and studied, as witnessed by many publications [5-7]. The main idea of argumentationbased negotiation is the ability to support offers with justifications and explanations, which play a key role in the negotiation settings. So, it allows the participants to the negotiation not only to exchange offers, but also reasons and justifications that support these offers in order to mutually influence their preference relations on the set of offers, and consequently the outcome of the dialogue.

It is simple to understand the parallelism between this approach and group decision-making. The idea of a group of agents exchanging arguments in order to achieve, for instance, a consensus, in order to support groups in decision-making process is easy to understand [8]. However the complexity of this process must not be underestimated, if considering a scenario where an agent seeks to defend the interests of who it represents and at the same time be part of a group that aims to reach a collective decision towards a problem for their organization $[9,10]$. Not only are those agents simultaneously competitive and cooperative but also represent human beings. Establishing some sort of dialog, as well as the different types of arguments that can be exchanged by agents is only the first step towards the problem resolution. One agent that represents a decision-maker involved in a process of group decision-making may show different levels of experience and knowledge related with the situation and should behave accordingly. Literature shows that there are works on the subject [1013], however it should be noted the existence of some flaws in terms of real world applicability of certain models. Some require high configuration costs that will not suit the different types of users they are built for and others show flaws that in our opinion are enough to affect the success of a Group Decision Support System (GDSS).

In this work it will be presented the most relevant models that allow inferring or configuring a behaviour style for a group decision-making context. It is also proposed a set of rules for which a behaviour model must follow without jeopardizing the entire GDSS and finally it is proposed an approach made through the modification of one existing model to the context of GDSS.

The rest of the paper is organized as follows: in the next section is presented the literature review. Section 3 presents our approach, where we identify different types of behaviours, defined with the use of an existing model and presented the set of rules 
that we believe that are the most important to allow defining types of behaviours for the agents in a way that does not compromise the system. In section 4 it will be discussed and debated how our approach can be applied to the context of GDSS and its differences compared with other existing approaches. Finally, some conclusions are taken in section 5, along with the work to be done hereafter.

\section{Literature Review}

The concern for identifying and understanding particular behavioural attitudes has led to many investigations and studies throughout the last decades with emphasis on proposing models and behaviour styles that can relate to the personality of the negotiator.

Carl Jung (1921), was the first to specify a model to study different psychological personality types based on four types of consciousness (sensation, intuition, thinking and feeling) that could in turn be combined with two types of attitudes (extraversion and introversion) and that way identify eight primary psychological types [14].

In 1962, Myers Briggs, developed a personality indicator model (The MeyersBriggs Type Indicator) based on Jung's theories [15]. This indicator is used as a psychometric questionnaire and allows people to understand the world around them and how they behave and make decisions based on their preferences [16]. This model was useful in order to identify different styles of leadership, which were later specified in Keirsey and Bate's publication [17], in 1984, as four styles of leadership:

- Stabilizer: tends to be very clear and precise when defining objectives and organizing and planning tasks in order to achieve them. Stabilizer leaders are also reliable and trustworthy due to the fact they show concern for other worker's necessities and problems. They are able to increase the motivation of their workers by setting tradition and organization as an example of success;

- Catalyst: the main focus is to develop the quality of own work and the one provided by their staff. They serve the facilitator's role by bringing the best out of other people, and motivate other workers with their own enthusiasm and potential;

- Trouble-shooter: as the name suggests, focus on dealing and solving problems. They show great aptitude for solving urgent problems by being practical and immediate. They bring people together as a team by analysing what needs to be done and informing exactly what to do as quickly as possible;

- Visionary: visionaries act based on their own intuition and perception of the problems in order to make decisions. They have a mind projected for the future and plan idealistic scenarios and objectives which may not always be achievable.

Related to vocational behaviour, Holland [18], in 1973 proposed a hexagonal model (RIASEC model) where he differentiates six types of personality mainly used in careers environments and to guide through the individual's choice of vocation. Those types are defined as:

- Realistic: realistic individuals value things over people and ideas. They are mechanical and athletic, and prefer working outdoors with tools and objects; 
- Investigative: investigative individuals have excellent analytic skills. They prefer working alone and solving complex problems;

- Artistic: artistic individuals show a deep sense of creativity and imagination. They prefer working on original projects and value ideas over things;

- Social: social individuals have high social aptitude, preferring social relationships and helping other people solving their problems. They prefer working with people over things;

- Enterprising: enterprising individuals show great communication and leadership skills, and are usually concerned about establishing direct influence on other people. They prefer dealing with people and ideas over things;

- Conventional: conventional individuals value order and efficiency. They show administrative and organization skills. They prefer dealing with numbers and words over people and ideas.

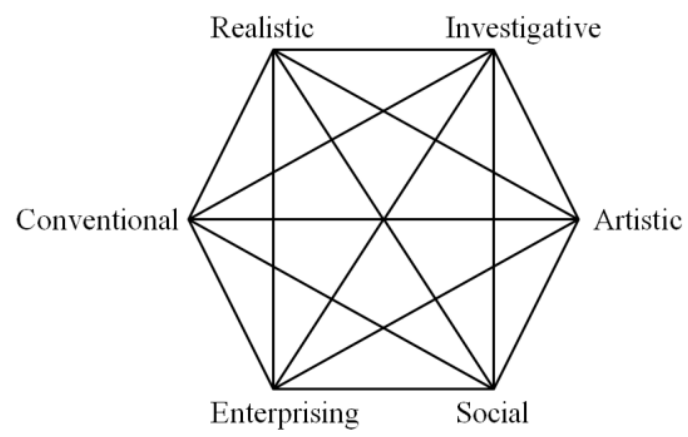

Fig. 1. Representation of Holland's hexagon model, adapted from [18]

It is important to note the distribution of these personalities in Holland's hexagon where personalities next to each other are the most similar while personalities facing against each other are the least similar (see Fig. 1).

Conflict management has always been an important area of decision-making, since it is very rare to find situations in group discussion where conflict is not present. In 1975, Thomas and Kilmann [19], also based on Jung's studies and a conflict-handling mode proposed by Blake and Mouton [20], suggested a model for interpersonal conflict-handling behaviour, defining five modes: competing, collaborating, compromising, avoiding and accommodating, according to two dimensions: assertiveness and cooperativeness. As seen in Fig. 2, both assertiveness and cooperativeness dimensions are related to integrative and distributive dimensions which were discussed by Walton and McKersie in 1965 [21]. Integrative dimensions refer to the overall satisfaction of the group involved in the discussion while distributive dimension refers to the individual satisfaction within the group. It is possible to see that the thinking-feeling dimension maps onto the distributive dimension while the introversion-extraversion dimension maps onto the integrative dimension. It is easy to understand this association by looking at competitors as the ones who seek the highest individual satisfaction, collaborators as the ones who prefer the highest satisfaction of the entire group. 
On the other hand avoiders do not worry about group satisfaction and accommodators do not worry about individual satisfaction. They also concluded that the thinkingfeeling dimension did not move towards the integrative dimension, and also that the introversion-extraversion did not move towards the distributive dimension.

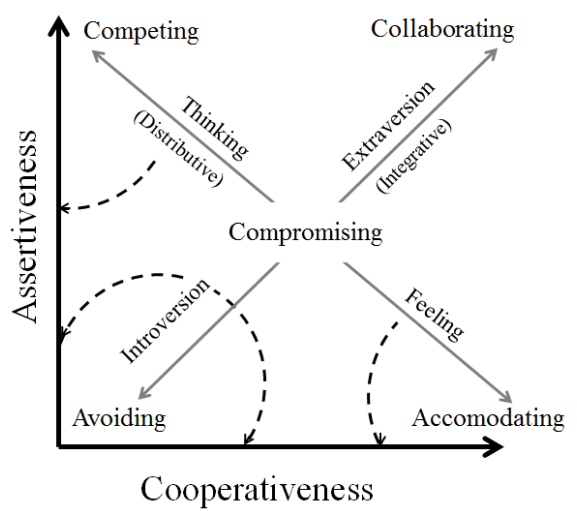

Fig. 2. Thomas and Kilmann's model for interpersonal conflict-handling behaviour, adapted from [19]

In 1992, Costa and McCrae [22] proposed a set of thirty traits extending the fivefactor model of personality (OCEAN model) which included six facets for each of the factors. These traits were used in a study made by Howard and Howard [23] in order to help them separate different kinds of behaviour styles and identify corresponding themes. A theme is defined as "a trait which is attributable to the combined effect of two or more separate traits". Those styles and themes are based on common sense and general research, and some of them have already been mentioned before in this literature review, however it is also important to referrer other relevant styles that were suggested such as the Decision and Learning styles. Decision style includes the Autocratic, Bureaucratic, Diplomat and Consensus themes while Learning style includes the Classroom, Tutorial, Correspondence and Independent themes.

In 1995, Rahim and Magner [24] created a meta-model of styles for handling interpersonal conflict based on two dimensions: concern for self and concern for the other. This was the base for the five management styles identified as obliging, avoiding, dominating, integrating and compromising as will be explained in detail in the Section 3.

\section{Methods}

It is really important to define correctly the agent's behaviour in order to not jeopardize the validation of the entire GDSS. Sometimes, in this area of research, there is an exhaustive concern to find a better result and because of that, other variables may be forgotten which can make impossible the use of a certain approach in those situations. 
For example: Does it make sense for a decision-maker or a manager from a large company, with his super busy schedule have the patience/time to answer (seriously) to a questionnaire of 44 questions like "the Big Five Inventory" so that he can model his agent with his personality? Due to reasons like this we have defined a list with considerations to have when defining types of behaviours for the agents in the context here presented. The definition of behaviour should:

1. Enhance the capabilities of the agents, i.e., make the process more intelligent, more human and less sequential, even though it may not be visible in the conceptual model it must not be possible for the programmer to anticipate the sequence of interactions just by reading the code;

2. Be easy to configure (usability) or not need any configuration at all from the user (decision-maker);

3. Represent the interests of the decision-makers (strategy used), so that agent's way of acting meets the interests defined by the user (whenever possible);

4. Not be the reason for the decision-makers to give up using the application, i.e., in a hypothetical situation, a decision-maker should not "win" more decisions just because he knows how to manipulate/configure better the system;

5. Be available for everyone to benefit from it. Obviously all decision-makers face meetings in different ways. Their interests and knowledge for each topic is not always the same. Sometimes it may be of their interest to let others speak first and only after gathering all the information, elaborate a final opinion on the matter. Other times it may be important to control the entire conversation and try to convince the other participants to accept out opinion straightaway.

By taking into account all these points, we propose in this article a behaviour model for the decision-making context based on conflict styles defined by Rahim and Magner (1995) [24]. The styles defined are presented in Fig. 3 and have been adapted to our problem. Rahim and Magner reckons the existence of 5 types of conflict styles: integrating, obliging, dominating, avoiding and compromising. In their work, they suggested these styles in particular to describe different ways of behave in conflict situations. They defined these styles according to the level of concern a person has for reaching its own goal and reaching other people's objectives. This definition goes along exactly with what we consider that the agents that operate in a GDSS context should be, when we say that they are both cooperative and competitive simultaneously. Therefore this model ends up describing 5 conflict styles which support what we think that is required for the agents to have a positive behaviour in this context. It also has the advantage of being a model easy to understand and to use.

In our approach, the configuration of agent's behavior made by the decisionmaker, will be done through the selection of one conflict style. The main idea is to define the agent with the participant's interests and strategies. For that, the definition of each conflict style should be clear and understandable for the decision-maker. The decision-maker can define in his agent different conflict styles throughout the process. For example, a decision-maker who is included in a decision process and has few or even no knowledge about the problem during the early stage of discussion. For that situation he may prefer to use an "avoiding" style and learn with what other people 
say, gather arguments and information that will support different options and that way learn more about the problem. In a following stage, when the decision-maker already has more information and knowledge about the problem, he may opt to use a more active and dominating style in order to convince others towards his opinion. Like mentioned before, there are many factors that can make the decision-maker face a meeting in different ways: interest about a topic, lack of knowledge about a topic, reckons the participation of more experienced people in the discussion, etc.

\section{Concern for Self}

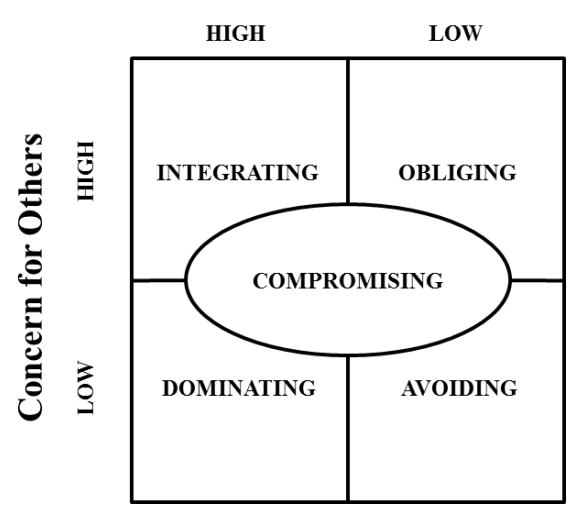

Fig. 3. Conflict Style, adapted from [24]

The different types of behaviour defined and that can be used by the agents are:

- Integrating (IN): This style should be selected every time the decision-maker considers that satisfying his own objectives is as important as satisfying the other participants' objectives. By choosing this conflict style, the agent will seek and cooperate with other agents in order to find a solution that is satisfactory to all the participants;

- Obliging (OB): This style should be selected if the decision-maker prefers to satisfy other participant's objectives instead of satisfying his own objectives. For example, in a situation where the decision-maker does not have any knowledge about the discussion topic;

- Dominating (DO): This style should be selected when the decision-maker only wants to pursuit his own objectives and do everything in his power to achieve them. For example, in a situation where the decision-maker is absolutely sure that his option to solve the problem is the most benefic. By using this style, the agent will be more dominant and will try to persuade the maximum possible agents. With this style the agent will prefer to risk everything to achieve his objectives even if that means he might end up at disadvantage because of that;

- Avoiding (AV): This style should be selected when the decision-maker does not have any interest in achieving either his own and other participants' objectives. For 
example, when the decision-maker has been include in a group discussion for which he does not have any sort of interest;

- Compromising (CO): This style should be selected when the decision-maker has a moderate interest in the topic and at the same time he also has a certain interest to achieve his own and other participants' objectives.

\section{Discussion}

Many approaches have been suggested in the literature which define/model agents with characteristics that will differentiate them from each other and as result will also show different ways of operating [11-13, 25-27]. However, even if many of those publications might be interesting for an academic context, they still show some issues that must be addressed. These issues that we will analyze are related to the context of support to group decision-making and also to competitive agents which that represent real individuals. There are several approaches in literature for (1) agents that are modeled according to the real participant personality (decision-maker) which they represent and (2) modeled with different intelligence levels (abilities) $[10,12]$. One of the most used technics in literature is "The Big Five Inventory" questionnaire that allows to obtain values for each one of the personality traits defined in the model of "The Big Five" (openness, conscientiousness, extraversion, agreeableness and neuroticism) [11, $26,28]$. Theoretically, we can think that the way agents operate, which is similar to real participant because it is modeled with "the same personality" is perfect. However, defining an agent with a conflict style based on the values of personality traits may not be the right way to identify the decision-maker. What makes a human act in a particular way is the result of much more than just its personality, it is a set of factors such as: personality, emotions, humor, knowledge, and body (physic part), and it can also be considered other factors such as sensations and the spiritual part [29]. Another relevant question is the fact this type of approach allows that certain agents have advantage over other agents. Many may say and think that this occurrence is correct, because close to what happens in real life, there are decision-makers that are more apt and therefore have advantage over other decision-makers. However the questions that arise are the following: Would a product like this used by decision-makers that knew they would be at disadvantage by using this tool? Would it be possible to sell a product that does not guarantee equality between its future users? It is also important to discuss another relevant analysis point which is the fact that this type of approach, in some situations, might provide less intelligent and more sequential outputs.

The study of different types of behaviour in agents has been represented in literature by a reasonable number of contributions. However, it is a subject that most of the times offers validation problems. Although there are proposals with cases of study aiming to validate this subject, that validation is somewhat subjective most of the times. Even when trying to mathematically formulate the problem so that it becomes scientifically "proven", that proof may often feel forced. A reflection of this problem is the difference between the practiced approaches for social and exact sciences. It is clear for us, as computer science researchers that it is not our goal to elaborate a mod- 
el for behavioural definition to use in specific scenarios. Instead, we will use a model defined and theoretically validated by others who work in areas that allow them to have these skills. However, the inclusion of intelligence in certain systems is growing at a blistering pace and some of the systems would not make sense nor would succeed without this inclusion. This means that it become more of common practice to adapt certain models that have not been designed specifically for the context for which they will be used. Because of that the evolution of the presented approaches will happen in an empirical way.

Another relevant condition is related with how most of the works are focused on very specific topics which may prevent a more pragmatic comparison of the various approaches. Even if in some situations the use of a specific technic (such as "The Big Five Inventory") might make sense, in others, and even though it may scientifically provide a case of study with brilliant results, it can be responsible for jeopardizing the success of the system. Our work aims to support each participant (decision-maker) in the process of group decision-making. It is especially targeted for decision support in ubiquitous scenarios where participants are considered people with a very fast pace of life, where every second counts (top managers and executives). In our context the system will notify the participant whenever he is added in a decision process (for instance, by email), and after that every participant can access the system and model his agent according to his preferences (alternatives and attributes classification), as well as how he plans to face that decision process (informing the agent about the type of behaviour to have), always knowing that there are no required fields in the agent setup. This way provides more freedom for the user to configure (depending on his interest and time) his agent with detail or with no detail at all. As can be seen in this context (and referred previously) the agents must be cooperative and competitive. They are cooperative because they all seek one solution for the organization they belong to, and competitive because each agent seeks to defend the interests of its participant and persuade other agents to accept his preferred alternative. For us this means that if an agent is both cooperative and competitive then it cannot exhibit behaviour where it is only concerned in achieving its objectives and vice versa.

\section{$5 \quad$ Conclusions and Future Work}

The use of agents to represent/support humans as well as their intentions in negotiation context is relatively common practice in literature. There are several approaches which based on relationships allow agents to judge different levels for trust, credibility, intelligence, etc. Specifically looking at support to group decision-making context, a few approaches have appeared and propose modelling agents based on a number of characteristics that will allow them to operate in a way similar to how the decision-maker would in real life. If in one hand the modelling of an agent with certain human characteristics makes sense since it allows to define different types of behaviours and strategies according to the objectives of the decision-maker, on the other hand even if some of those approaches may seem intellectually interesting and complex, they affect the system where they belong due to many reasons, as for instance: 
illusory intelligence creation, unbalanced agent capabilities, high configuration costs e and weak representation of what in practice the decision-maker would want the agent operating model to be.

In this paper we presented (1) a study about the most important models that can be used to infer different types of behaviours that can be adapted and used in this context, (2), a set of rules that must be followed and that will positively affect the system when defining behaviours and (3) is proposed the adaptation of a conflict management model in the context of GDSS. Furthermore we included a new approach of how to look at this problem, and alert to the negative impact some other approaches might have in the system where they are used. Our approach intends to provide a more perceptible and concrete way for the decision-maker to understand the five types of behaviour that can be used to model the agent in support to group decision-making context where each agent represents a decision-maker. We believe that with our approach it will be simpler for agents to reach or suggest solutions since they are modeled with behaviours according to what the decision-maker wants. This makes it easier to reflect in the agent the concern to achieve the decision-maker's objectives or the objectives belonging to other participants in the decision process. With this approach the agents follow one defined type of behaviour that also works as a strategy that can be adopted by each one of the decision-makers.

As for future work we will work in the specific definition of each type of behaviour identified in this work. We intend to describe behaviours according to certain facets proposed in the Five Factor Model and also study tendencies for each type of behaviour to make questions, statements, and requests. At later stage we will integrate this model in the prototype of a group decision support system which we are developing.

\section{Acknowledgements}

This work is part-funded by ERDF - European Regional Development Fund through the COMPETE Programme (operational programme for competitiveness) within project FCOMP-01-0124-FEDER-028980 (PTDC/EEISII/1386/2012) and by National Funds through the FCT - Fundação para a Ciência e a Tecnologia (Portuguese Foundation for Science and Technology) with the João Carneiro PhD grant with the reference SFRH/BD/89697/2012.

\section{References}

1. Rahwan, I., Ramchurn, S.D., Jennings, N.R., Mcburney, P., Parsons, S., Sonenberg, L.: Argumentation-based negotiation. The Knowledge Engineering Review 18, 343-375 (2003)

2. Hadidi, N., Dimopoulos, Y., Moraitis, P.: Argumentative alternating offers. Argumentation in Multi-Agent Systems, pp. 105-122. Springer (2011) 
3. El-Sisi, A.B., Mousa, H.M.: Argumentation based negotiation in multiagent system. In: Computer Engineering \& Systems (ICCES), 2012 Seventh International Conference on, pp. 261-266. IEEE, (1012)

4. Marey, O., Bentahar, J., Asl, E.K., Mbarki, M., Dssouli, R.: Agents' Uncertainty in Argumentation-based Negotiation: Classification and Implementation. Procedia Computer Science 32, 61-68 (2014)

5. Mbarki, M., Bentahar, J., Moulin, B.: Specification and complexity of strategic-based reasoning using argumentation. Argumentation in multi-agent systems, pp. 142-160. Springer (2007)

6. Amgoud, L., Vesic, S.: A formal analysis of the outcomes of argumentation-based negotiations. In: The 10th International Conference on Autonomous Agents and Multiagent Systems-Volume 3, pp. 1237-1238. International Foundation for Autonomous Agents and Multiagent Systems, (2011)

7. Bonzon, E., Dimopoulos, Y., Moraitis, P.: Knowing each other in argumentation-based negotiation. In: Proceedings of the 11th International Conference on Autonomous Agents and Multiagent Systems-Volume 3, pp. 1413-1414. International Foundation for Autonomous Agents and Multiagent Systems, (2012)

8. Kraus, S., Sycara, K., Evenchik, A.: Reaching agreements through argumentation: a logical model and implementation. Artificial Intelligence 104, 1-69 (1998)

9. Faratin, P., Sierra, C., Jennings, N.R.: Negotiation decision functions for autonomous agents. Robotics and Autonomous Systems 24, 159-182 (1998)

10. Rahwan, I., Kowalczyk, R., Pham, H.H.: Intelligent agents for automated one-to-many ecommerce negotiation. In: Australian Computer Science Communications, pp. 197-204. Australian Computer Society, Inc., (2002)

11. Santos, R., Marreiros, G., Ramos, C., Neves, J., Bulas-Cruz, J.: Personality, emotion, and mood in agent-based group decision making. (2011)

12. Kakas, A., Moraitis, P.: Argumentation based decision making for autonomous agents. In: Proceedings of the second international joint conference on Autonomous agents and multiagent systems, pp. 883-890. ACM, (2003)

13. Zamfirescu, C.-B.: An agent-oriented approach for supporting Self-facilitation for group decisions. Studies in Informatics and control 12, 137-148 (2003)

14. Jung, C.G.: Psychological types, volume 6 of The collected works of CG Jung. Princeton University Press 18, 169-170 (1971)

15. Myers-Briggs, I.: The Myers-Briggs type indicator manual. Prinecton, NJ: Educational Testing Service (1962)

16. Myers, I.B., Myers, P.B.: Gifts differing: Understanding personality type. Davies-Black Pub. (1980)

17. Bates, M., Keirsey, D.: Please Understand Me: Character and Temperament Types. Del Mar: Prometheus Nemesis Book Co (1984)

18. Holland, J.L.: Making vocational choices: A theory of vocational personalities and work environments. Psychological Assessment Resources (1997)

19. Kilmann, R.H., Thomas, K.W.: Interpersonal conflict-handling behavior as reflections of Jungian personality dimensions. Psychological reports 37, 971-980 (1975)

20. Blake, R.R., Mouton, J.S.: The new managerial grid: strategic new insights into a proven system for increasing organization productivity and individual effectiveness, plus a revealing examination of how your managerial style can affect your mental and physical health. Gulf Pub. Co. (1964)

21. Walton, R.E., McKersie, R.B.: A behavioral theory of labor negotiations: An analysis of a social interaction system. Cornell University Press (1991) 
22. Costa, P.T., MacCrae, R.R.: Revised NEO Personality Inventory (NEO PI-R) and NEO Five-Factor Inventory (NEO FFI): Professional Manual. Psychological Assessment Resources (1992)

23. Howard, P.J., Howard, J.M.: The big five quickstart: An introduction to the five-factor model of personality for human resource professionals. ERIC Clearinghouse (1995)

24. Rahim, M.A., Magner, N.R.: Confirmatory factor analysis of the styles of handling interpersonal conflict: First-order factor model and its invariance across groups. Journal of Applied Psychology 80, 122 (1995)

25. Allbeck, J., Badler, N.: Toward representing agent behaviors modified by personality and emotion. Embodied Conversational Agents at AAMAS 2, 15-19 (2002)

26. Badler, N., Allbeck, J., Zhao, L., Byun, M.: Representing and parameterizing agent behaviors. In: Computer Animation, 2002. Proceedings of, pp. 133-143. IEEE, (2002)

27. Velásquez, J.D.: Modeling emotions and other motivations in synthetic agents. In: AAAI/IAAI, pp. 10-15. Citeseer, (1997)

28. Durupinar, F., Allbeck, J., Pelechano, N., Badler, N.: Creating crowd variation with the ocean personality model. In: Proceedings of the 7th international joint conference on Autonomous agents and multiagent systems-Volume 3, pp. 1217-1220. International Foundation for Autonomous Agents and Multiagent Systems, (2008)

29. Pasquali, L.: Os tipos humanos: A teoria da personalidade. differences 7, 359-378 (2000) 